\title{
A New Method of Image Fusion Technique for Impulse Noise Removal in Digital Images using the quality Assessment in Spatial Domain
}

\author{
Ajay kumar Beesetti ${ }^{1}$, Dr.Rajyalakshmi Valluri ${ }^{2}$, Minusha Songa ${ }^{3}$ \\ ${ }^{I}$ Ajay Kumar Beesetti, B.E Student, Department of ECE, ANITS/Andhra University(A.U), Visakhapatnam. \\ ${ }^{2}$ Dr.Rajyalakshmi Valluri, Associate Professor, Department of ECE, ANITS/A.U, Visakhapatnam. \\ ${ }^{3}$ Minusha Songa, B.Tech Student, Department of ECE,SPMV, Tirupati.
}

\begin{abstract}
Due to advancement of technology, remote sensing plays very important role in satellite based communication. Satellite gives images in digital format. The digital images are corrupted by impulse noise due to errors generated in camera sensors, analog-to-digital conversion and communication channels. The noise density varies depending on various factors namely reflective surfaces, atmospheric variations, noisy communication channels etc. Impulse noise corruption is very common in digital images. Therefore it is necessary to remove impulse noise in-order to provide further processing such as edge detection, segmentation, pattern recognition etc. Filtering a noisy image, while preserving the image details is one of the most important issues in image processing. The images captured by different sensors, producing different impulse noisy images are considered and they undergoes iterative filtering algorithm, search for the noise-free pixels within a small neighborhood. These filtered images are combined to a single image called image fusion, which retains the important features of the images from individual sensors. In this paper, we introduce an image fusion technique for impulse noise reduction, where the fused image will combine the uncorrupted pixels of the filtered noisy images. The performance of the Image Fusion is evaluated by using a reference image quality metric, Structural similarity Index (SSIM), to estimate how well the important information in the de-noised images is represented by the fused image. Experimental results show that the fused image has more quality than other filtered images.
\end{abstract}

Keywords - Impulse Noise, Image fusion, Filtering, De-noised Image.

\section{Introduction}

Digital images are often corrupted during acquisition, transmission or due to faulty memory locations in hardware [1]. The impulse noise can be caused by a camera due to the faulty nature of the sensor or during transmission of coded images in a noisy communication channel [2]. Noise is undesired information that contaminates an image. Noise appears in image from various sources. The digital image acquisition process converts an optical image into a continuous electrical signal. This electrical signal sampled, is primary process by which noise appears in digital image. There are several ways through which noise can be introduced into an image, depending on how the image is created. This is the main problem in remote sensing applications. In this paper, the images captured by five different sensors are filtered using five different non-linear filtering algorithms such as Standard Vector Median Filter (VMF), Rank Conditioned VMF, Rank conditioned and Threshold VMF, Center Weighted VMF and Absolute Deviation VMF., producing five de-noised images. These de-noised images are fused using our fusion technique, thus obtaining a high quality image.

\section{Impulse Noise In Digital Images}

Impulse noise is independent and uncorrelated to the image pixels and is randomly distributed over the image. For an impulse noise corrupted image all the image pixels are not noisy, a number of image pixels will be noisy and the rest of pixels will be noise free. There are different types of impulse noise namely salt and pepper type of noise and random valued impulse noise. In salt and pepper type of noise the noisy pixels takes either salt value (gray level -225) or pepper value (grey level -0) and it appears as black and white spots on the images. In case of random valued impulse noise, noise can take any gray level value from zero to 225 . In this case also noise is randomly distributed over the entire image and probability of occurrence of any gray level value as noise will be same.

\section{Order Static Filters For Color Image Processing}

Order-static filters are nonlinear filters whose response is based on the ordering (ranking) the pixels contained in the image area encompassed by the filter, and then replacing the value of the center pixel with the value determined by the ranking result. 
A. Vector Median Filter:

In the vector median filter for the ordering of vectors in a particular kernel or mask a suitable distance measure is chosen. The vector pixels in the window are ordered on the basis of the sum of distances between each vector pixel and the other pixels in the window. The sum of the distances is arranged in ascending order and then the same ordering is associated with vector pixels. The vector pixel with the smallest distances is the vector median pixel. The vector median filter is represented as

$$
X_{V M F}=\text { vector median (window) }
$$

If $\delta_{\mathrm{i}}$ is the sum of the distances of the $\mathrm{i}^{\text {th }}$ vector pixel with all the other vectors in the kernel, then

$$
\delta_{i}=\sum_{j=1}^{n} \Delta\left(x_{i}, x_{j}\right)
$$

where $(1 \leq \mathrm{i} \leq \mathrm{N})$ and $\mathrm{X}_{\mathrm{i}}$ and $\mathrm{X}_{\mathrm{j}}$ are the vectors, $\mathrm{N}=9 . \Delta\left(\mathrm{X}_{\mathrm{i}}, \mathrm{X}_{\mathrm{j}}\right)$ is the distance measure given by the $\mathrm{L} 1 \mathrm{norm}$ or the city block distance which is more suited to non correlated noise. The ordering may be illustrated as

$$
\delta_{1} \leq \delta_{2} \leq \delta_{3} \leq \ldots \leq \delta_{9}
$$

and this implies the same ordering to the corresponding vector pixels

$$
\text { i.e., } \mathrm{X}(1) \leq \mathrm{X}(2) \leq, \ldots, \leq \mathrm{X}(9)
$$

where the subscripts are the ranks. Since the vector pixel with the smallest sum of distances is the vector median pixel, it will correspond to rank 1 of the ordered pixels,

\section{B. Median Filter:}

$$
\text { i.e., } \quad \mathrm{X}_{\mathrm{VMF}}=\mathrm{X}(1)
$$

The Median Filter [8] as the name implies, replaces the value of the pixel by the median of the intensity values in the neighborhood of that pixel defined in (1). The pixel with the median magnitude is used to replace the pixel in the signal studied.

$$
\text { i.e.; Median Filter }\left(\mathrm{x}_{1}, \mathrm{x} 2, \ldots \mathrm{xN}\right)=\operatorname{Median}\left(\mathrm{x}_{1}, \mathrm{x}_{2}, \ldots \ldots, \mathrm{x}_{\mathrm{N}}\right)
$$

The median filter is more robust with respect to the presence of noise.

C. Center Weighted Vector Median Filter:

In the case of center weighted median filter the kernel vector pixels are assigned some non negative values called weights. The central vector pixel is assigned a non negative weight while the weight of the neighboring pixels is kept unity. The weights denote the number of copies is obtained. The output $\mathrm{Y}$ (say), of a weighted median filter of span $\mathrm{N}$ (where $\mathrm{N}$ generally denotes the kernel size, $\mathrm{N}=9$ ) associated with $\mathrm{N}$ integer weights,

$$
\mathrm{W}=[\mathrm{W} 1 . \mathrm{W} 2 \ldots . \mathrm{WN}]
$$

$$
\mathrm{Y}=\text { vector median }[\mathrm{W} 1 * \mathrm{X} 1, \mathrm{~W} 2 * \mathrm{X} 2, \ldots ., \mathrm{W} 9 * \mathrm{X} 9]
$$

Where the vector median [ ] denotes the vector median operation. The center weight is kept odd so that a central element is always obtained. If the center weight has value of one then the present filter becomes a vector median filter. If the center weight is given a value greater that the kernel size then the filter performs as an identity filter. Larger central vector weights imply superior detail preservation and the inferior noise removal capability as compared to smaller central weights.

D. Spatial Median Filter:

The Spatial Median Filter (SMF) [5] is a uniform smoothing algorithm with the purpose of removing noise and fine points of image data while maintaining edges around larger shapes. The SMF is based on the spatial median quintile function which is a L1 norm metric that measures the difference between two vectors. The spatial depth between a point and a set of points is defined by

$$
\boldsymbol{S}_{\text {Dept } h}\left(X, X_{1}, X_{2}, X_{3, \ldots \ldots . . .} X_{N}\right)=1-\frac{1}{N-1}\left\|\sum_{i=1}^{N} \frac{X-x_{i}}{\left\|X-x_{i}\right\|}\right\|
$$

Let $\mathrm{r}_{1}, \mathrm{r}_{2}, \ldots, \mathrm{r}_{\mathrm{N}}$ represent $\mathrm{X}_{1}, \mathrm{X}_{2}, \ldots . . \mathrm{X}_{N}$ in rank order such that

$$
\begin{aligned}
& \geq S_{\text {Depth }}\left(r_{1}, X_{1}, X_{2}, \ldots \ldots . X_{N}\right) \\
& \geq S_{\text {Depth }}\left(r_{2}, X_{1}, X_{2}, \ldots \ldots . X_{N}\right) \\
& \geq S_{\text {Depth }}\left(r_{N}, X_{1}, X_{2}, \ldots \ldots . X_{N}\right)
\end{aligned}
$$

and let $r_{c}$ represent the center pixel under the mask.

Then $\operatorname{SMF}\left(\mathrm{x}_{1}, \mathrm{x}_{2}, \ldots \ldots \mathrm{x}_{\mathrm{n}}\right)=\mathrm{r}_{1}$

\section{Multi Sensor Image Fusion Using Qualitative Assessment}

Given five de-noised images, it is required to combine the images into a single one that has all objects without producing details that are non-existent in the given images. Here $\mathrm{R}^{1}$ is VMF median filtered image, $\mathrm{R}^{2}$ is median filtered image, $R^{3}$ is the CMF filtered image, $R^{4}$ is the SMF filtered image.

The fusion algorithm consists of the following steps:

a. Input images $R_{i}$ for $i=1,2,3,4$ are divided into non-overlapping rectangular blocks with size of mxn (10x10 blocks). The $j^{\text {th }}$ image blocks of $R^{i}$ are referred by $R_{j}^{i}$ 
b.Variance (VAR) of $\mathrm{R}_{\mathrm{j}}^{\mathrm{i}}$ are calculated for determining the sharpness values of the corresponding blocks and the results of $\mathrm{R}_{\mathrm{j}}^{\mathrm{i}}$ are denoted by $\mathrm{VAR}_{\mathrm{j}}^{\mathrm{i}}$. VAR is defined as:

Where $\bar{f}$ is the average grey level over the image.

$$
V A R=\frac{1}{m \times n} \sum_{x} \sum_{y}(f(x, y)-\bar{f})
$$

$$
\bar{f}=\frac{1}{m \times n} \sum_{x} \sum_{y} f(x, y)
$$

c. In order to determine the sharper image block, the variances of image blocks from five images are sorted in descending order and the same ordering is associated with image blocks. The block with the maximum variance is kept in the fused image. The fusion mechanism is represented as follows:

If $\operatorname{VAR}_{(\mathrm{k})}$ is the variance of block $\mathrm{R}_{\mathrm{j}}^{\mathrm{i}}$, where $\mathrm{k}$ denotes the rank, the ordering of variances is given by

and this implies the same ordering to the corresponding blocks

$$
\mathrm{VAR}_{(1)}>\mathrm{VAR}_{(2)}>\mathrm{VAR}_{(3)}>\mathrm{VAR}_{(4)}>\mathrm{VAR}_{(5)}
$$

$$
\mathrm{R}_{(1)}>\mathrm{R}_{(2)}>\mathrm{R}_{(3)}>\mathrm{R}_{(4)}>\mathrm{R}_{(5)}
$$

where the subscripts are the ranks of the image blocks. Since the block with the smallest variance is in the fused image, it will correspond to rank 1 of the ordered blocks

$$
\text { i.e. Fused Block }=\mathrm{R}_{(1)}
$$

\section{Qualitative Assessment Of Spatial Domain}

Quality assessment of images has been successfully employed in the authentication area, such as iris and fingerprint verification, which is used to evaluate the quality of the captured images. Fingerprint quality is usually defined as a measure of the clarity of the ridge and valley structures. In the multi-focus image fusion, it is the first step to investigate whether the region of the image is clarity or not, so we incorporate the quality assessment into multi-focus image fusion algorithm. Generally speaking, the quality assessment can be classified into two ways, namely the frequency domain assessment and the spatial domain assessment. We incorporate the latter one into this algorithm. In order to assess the image quality in a local region, we partition a given image into a lattice of blocks of size bxb. For each block B, let $g s=\left(g_{s}{ }^{x}, g_{s}{ }^{y}\right)$ denote the gradient of the gray level intensity at site $\mathrm{s} \in \mathrm{B}$. The covariance matrix of the gradient vectors for all $\mathrm{b}^{2}$ sites in

$$
J=\frac{1}{b^{2}} \sum_{s \in b} g_{s}^{T} g_{s}=\left[\begin{array}{ll}
j_{11} & j_{12} \\
j_{21} & j_{22}
\end{array}\right]
$$

The above symmetric matrix is positive semi definite with Eigen values

$$
\begin{aligned}
\gamma_{1} & =\frac{1}{2}(\operatorname{trace}(J)+\sqrt{\operatorname{trace}(J)-4 \operatorname{det}}(J) \\
\gamma_{2} & =\frac{1}{2}(\operatorname{trace}(J)-\sqrt{(\operatorname{trace}(J)-4 \operatorname{det})(J)}
\end{aligned}
$$

Where trace $(J)=\mathrm{j}_{11}+\mathrm{j}_{22}$, det $(\mathrm{J})=\mathrm{j}_{11} \mathrm{j}_{22}-\mathrm{j}_{12}{ }^{2}$ and $\gamma_{1} \geq \gamma_{2}$.

Quality assessment defined as

$$
\gamma=\gamma_{1}-\gamma_{2}
$$

This measure reflects the clarity of the local region. The clearer the local region is, the bigger the measure is

\section{Experimental Results}

The performance evaluation of filtering using image fusion method is tested on the true color remote sensing image with 290x290 pixels. The impulse noise is added into the image with different noise densities. Here we are assuming, $n=4$, i.e.; 4 sensors. The noisy image is processed using a iterative filtering algorithm based on the noise density in the image. These filtered images are fused into a single image using Quality Assessment of Spatial Domain. The experimental results are shown in Figure 1. Table (1) shows the results of PSNR values of fused image with different noise densities. We used the image quality metric, peak signal-tonoise ratio (PSNR), to measure the quality of the restored image. The PSNR measure is defined as

$$
P S N R=10 \log _{10} \frac{(255)^{2}}{\mathrm{MSE}}
$$

where MSE is the mean squared error between the original noise-free image and the restored image.

\begin{tabular}{lccc}
\hline \hline & SSIM & MSE & PSNR \\
\hline Fusion & 0.99 & 50 & 29 \\
VMF & 0.93 & 200 & 25 \\
Median Filter & 0.92 & 220 & 22 \\
Center Weighted Median Filter & 0.91 & 650 & 20 \\
Spatial Median Filter & 0.90 & 700 & 18
\end{tabular}

Table 1: Performance Evaluation of Fusion Method with filter responses at 30\% of noise intensity 


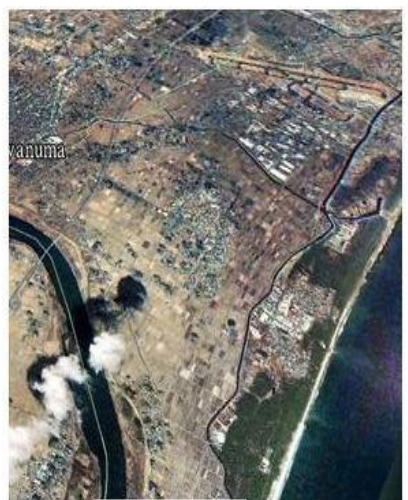

a) Original Image

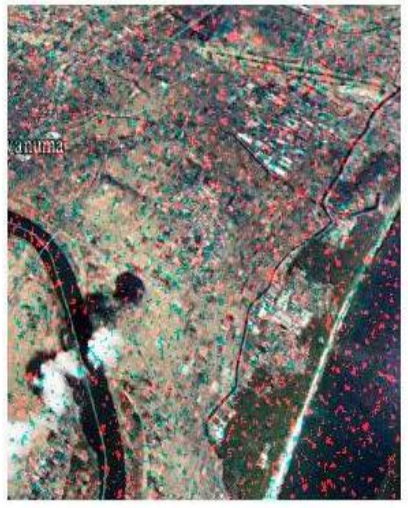

d) Center-Weighted Median Filter

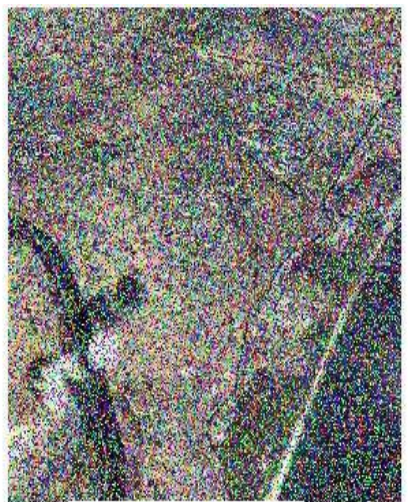

b) Image with added Noise

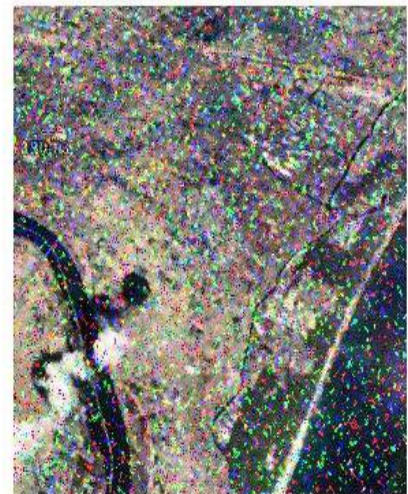

e) Spatial Median Filter

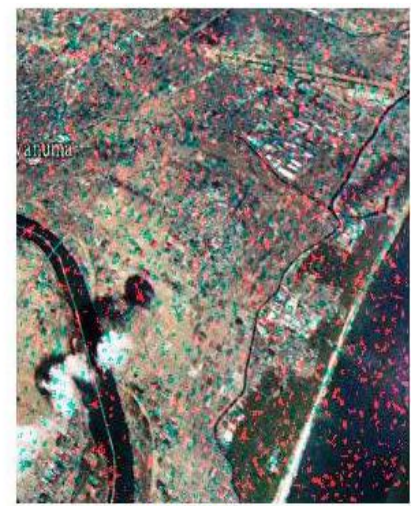

c) VMF Response

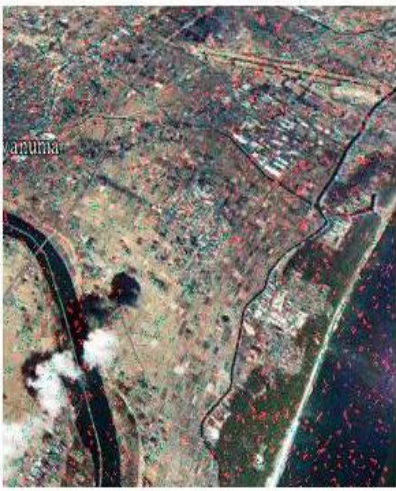

f) Fused Image

Fig 1: Fused Image and responses of different kinds of filters

a) Original image; b) Image with added Noise; c) Vector Median Filter; d) Center Weighted Median Filter;

e) Spatial Median Filter Image; f) Fused Image

\section{Conclusion}

This paper presents a new method of image fusion technique for removal of impulse noise in images. The images captured by sensors undergo filtering using VMF and SMF, and then the two individual de-noised images are fused to obtain a high quality image. The Quality of the images is evaluated using Structural Similarity Index (SSIM), Peak Signal-to-Noise ratio (PSNR) and Mean Square Error (MSE) with different noise densities. The proposed techniques are algorithmically simple and can be used for real time imaging applications.

\section{References}

[1] Nikolaos Mitianoudis, Tania Stathaki, Pixel-based and Region-based Image Fusion schemes using ICA bases, Communications and Signal Processing group, Preprint submitted to Elsevier Science, 13th Dec 2007.

[2]. Reihard Berstein, "Adaptive nonlinear filters for simultaneous removal of different kinds of noise in images," IEEE Trans on circuits and systems, Vol.cas-34, no11, pp.127-1291, Nivember1987.

[3] A. Hyv Äarinen, P. O. Hoyer, and E. Oja. Image denoising by sparse code shrinkage. In S. Haykin and B. Kosko, editors, Intelligent Signal Processing. IEEE Press, 2001.

[4] P. Hill, N. Canagarajah, and D. Bull. Image fusion using complex wavelets. In Proc.13th BritishMachineVisionConference, Cardi ${ }^{\circledR}, U K, 2002$

[5] James C. Church, Yixin Chen, and Stephen V. Rice, -A Spatial Median Filter for Noise Removal in Digital Images-, 2008 IEEE.

[6] M. Vett, erli and C. Herley, "Wavelets and filter banks: theory and design," IEEE Tram Signal Processing, vol. 40, pp. 2207-2232, September 1992.

[7] P. Burt and R. Lolczynski, "Enhanced image capture through fusion," in Proc. the Fourtt International Conference on Computer Vision, pp. 173- 182, 1993.

[8] Tao Chen, Kai-Kaung Ma and Li-Hui Chen, -"Tri-state median filter for image Denoising", IEEE Transactions on Image Processing, Vol 8, no.12, pp.1834-1838, December 1999.

[9] S.Indu, Chaveli Ramesh, _ "A noise fading technique for images corrupted with impulse noise", Proceedings of ICCTA07,IEEE. 